\title{
Atypical Anterior Uveitis in Behcet's Disease
}

\author{
Mehdi Khamaily, Imane Tarib, Joumany Brahim Salem, Sidi Dahi, Yassine Malek, Taoufik \\ Abdellaoui, Yassine Mouzari, Fouad El Asri, Karim Reda, Abdelbarre Oubaaz
}

\begin{abstract}
Behcet's disease (BD) is a chronic multisystem disorder,characterized by relapsing inflammation, Ocular involvement is frequent $(\mathbf{4 0 - 7 0 \% )}$, and all coats of the eye may be affected. The non-granulomatous character of uveitis is usually reported in BD.

We report a series of 11 cases of atypically granulomatous uveitis in patients with extra ocular signs of Behcet.
\end{abstract}

\section{Index Terms - Granulomatous uveitis, Behcet, atypical.}

\section{INTRODUCTION}

Behcet's disease (BD) is a chronic multisystem disorder,characterized by relapsing inflammation for wich the underllynig histopathology is an occlusive vasculitis, and whose etiology is still poorly known [1]. The exact etiopathogenesis of the disease is still unclear, although genetic predisposition, environmental factors, and immunologic abnormalities have been considered [2].

It is classically characterized by recurrent oral aphthae (the main and the most recurrent symptom), genital ulcerations, variable skin lesions, uveitis, and peripheral arthritis.

Ocular involvement is frequent (40-70\%), representing an important clinical criterion. Responsible for a serious attack, conditioning the visual prognosis.

All coats of the eye may be affected. The nongranulomatous character of uveitis is usually reported in $\mathrm{BD}$ [3].

\section{PURPOSE OF THE STUDY}

The purpose of this study is to report for the first time cases of granulomatous uveitis noted in patients with BD.

\section{PATIENTS AND METHODS}

Retrospective study, conducted between January 2010 and December 2019, at the Mohammed V Military Teaching Hospital

The diagnosis of $\mathrm{BD}$ was selected according to the

Published on July 14, 2020

Mehdi Khamaily, Mohammed V Military Hospital, Morocco.

(corresponding e-mail: mehdi.khamaily@gmail.com)

Imane Tarib, Mohammed V Military Hospital, Morocco.

Joumany Brahim Salem, Mohammed V Military Hospital, Morocco.

Sidi Dahi, Mohammed V Military Hospital, Morocco.

Yassine Malek, Mohammed V Military Hospital, Morocco.

Taoufik Abdellaoui, Mohammed V Military Hospital, Morocco.

Yassine Mouzari, Mohammed V Military Hospital, Morocco.

Fouad El Asri, Mohammed V Military Hospital, Morocco.

Karim Reda, Mohammed V Military Hospital, Morocco.

Abdelbarre Oubaaz, Mohammed V Military Hospital, Morocco. international study group for Behcet disease (1990).

Ocular involvement was defined by the presence of anterior, intermediate or posterior uveitis or pan-uveitis associated or not with a retinal vasculitis in the fundus of the eye or a retinal angiography.

An assessment has been carried out to eliminate any common cause of granulomatous uveitis: conversion enzyme, thoracic radio, TST (tuberculin skin test), infectious serologies, namely syphilitic and toxoplasmic, search for signs of Vogt Koyanagi Harada.

\section{RESULTS}

Among 277 cases of ocular involvement in BD, 11 cases of granulomatous uveitis were found. The sex ratio was 4.5 , with an average age at diagnosis of 30 years and an average age at onset of ocular symptomatology of 36 years.

It was inaugurated in two patients. Associated with parenchymal neurological involvement in two cases and vascular involvement with deep vein thrombosis in one case. The pathergy test was positive in nine cases.

Ocular involvement was bilateral in nine patients. The ophthalmologic examination revealed:

- $\quad$ Anterior uveitis in 2 patients

- A posterior uveitis in 4 patients

- $\quad$ Pan-uveitis in 5 patients

- $\quad$ Retinal vasculitis in 4 patients.

The granulomatous character was noted in the presence of ant eggs in six patients and mutton-fat precipitates behind Descemet's membrane in five patients.

we report a case from our series, this is a patient admitted for a table of granulomatous uveitis (Fig. 1) with extra ocular signs in favor of behcet's disease (Fig. 2).

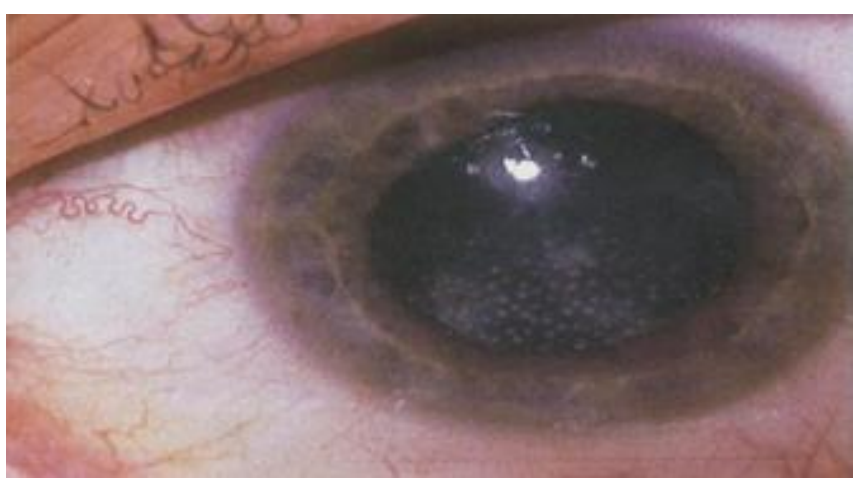

Fig. 1. Atypical Granulomatous Uveitis in 37-year-old patient with Behcet's Disease.

From a therapeutic point of view, all patients underwent full-dose corticosteroid therapy. An immunosuppressant type cyclophosphamide by bolus was introduced in 9 
patients, with a relay initiated in 8 patients by azathioprine. One of these patients was in addition treated with biotherapy (anti-TNF) due to severe ocular involvement. The average duration of follow-up was three years. A good response was noted in $64.4 \%$.

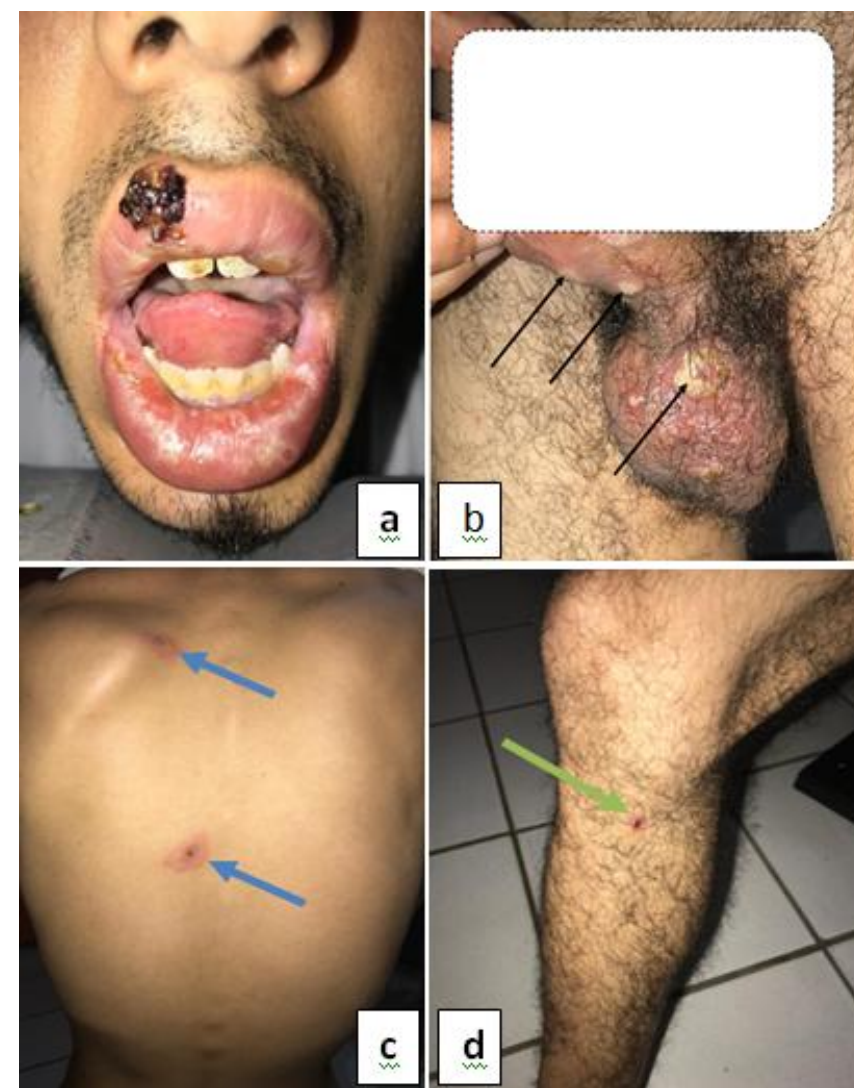

Fig.2. Extra ocular sign of Behcet's disease with atypical Granulomatous Uveitis in 37-year-old patient a) multiple oral aphthose;

b) multiple genital aphthoses (black arrow);

c) Papulopustular lesions in the back (blue arrow;

d) Papulopustular lesions in the leg (green arrow).

\section{DISCUSSION}

Ocular involvement in BD occurs in $67-95 \%$ of cases. It is often bilateral (63 to $100 \%$ of cases) [4].

The first inflammatory outbreaks are rather unilateral and anterior [5]. The recurrences will concern the posterior segment and become bilateral. Uveitis can be inaugural in 10 to $20 \%$ of cases, otherwise it appears 2 to 3 years after aphthuous ulcers [6].

The ocular involvement in the BD is very rich and protean, it touches all the tunics of the eye [7]. Several types of ocular involvement have been described, but the granulomatous character did not appear in the previous studies.

This series reports for the first time cases of granulomatous uveitis in patients with BD on a sufficient number of criteria. This may lead to not excluding the possibility of BD in the presence of granulomatous uveitis if the clinical context is suggestive, and after eliminating the usual causes of granulomatous uveitis. However, further studies are needed to substantiate these findings.

\section{CONCLUSION}

- Uveitis is the primary ocular involvement of Behcet's Disease.

- It is a part of the diagnostic criteria for the disease.

- Generally described as non-granulomatous

- $\quad$ First series having reported granulomatous uveitis in Behcet's disease, but a small series ...

In case of a suggestive clinical presentation, despite the granulomatous character of uveitis is suggestive, consider the possibility of Behcet's disease.

\section{REFERENCES}

[1] I. Ksiaa et al .Ey and Behcet disease.J Fr Ophtalmol.42(6) 626-641 (2019)

[2] Rokutanda R, Kishimoto M, Okada M. Update on the diagnosis and management of Behçet's disease. Open Access Rheumatol 2014;30:1-8.

[3] Z Hajji et al Encyclopédie Médico-Chirurgicale.21-225-C-20 (2010)

[4] Yohei Kirini et al Clinical and genetic Aspects of Behcet's disease in Japan .Intern Med 58(9) 1199-1207 (2019).

[5] Uwe Pleyer et al.Anterior uveitis clinic Diagnostic procedure and treatment options.Klin Monbl Augenheilkd (2020).

[6] Sinem Nihal Esatogln et al.Update on the treatmentof Behcet's syndrom.Intern Emerg Med 14,661-675 (2019)

[7] Jing Zhangetal. A review of behcet's disease from perspectives of both western and chinese medicine.J Tradit Chi Med39(1) ; 139-152 ( 2019). 